\title{
Gamma Heavy Chain Disease: 2 Cases
}

\author{
Ramasamy I** and Rudzki $\mathbf{Z}^{2}$ \\ 'Department of Biochemistry, Worcester Royal Hospital, \\ UK \\ ${ }^{2}$ Consultant Histopathologist, Heart of England NHS \\ Trust, UK \\ *Corresponding author: Ramasamy I, Department \\ of Biochemistry, Worcester Royal Hospital, UK; Email: \\ indrar@ozemail.com.au
}

Received: March 18, 2018; Accepted: April 17, 2018; Published: May 07, 2018

\begin{abstract}
Gamma heavy chain disease ( $\mathrm{gHCD}$ ) is a rare lymphoproliferative disorder characterized by the production of a truncated immunoglobulin heavy chain. Fewer than 200 cases have been reported in the literature. In some cases gHCD occurs with other lymphoid neoplasms. This study reports clinical, biochemical, haematological and histological findings in two cases gHCD. The first case is a 88 year old woman with gHCD. The second case is a 81 year old woman who developed gHCD during treatment for Waldenstrom'smacroglobulinemia. In the second patient histopathology identified a separate clone responsible for the secretion of gamma heavy chain. Studies on the clonal evolution of the disease may provide insight into therapeutic implications and the genomic complexity of the disease.
\end{abstract}

Keywords: Gamma heavy chain disease; Franklin's disease; Waldenstrom'smacroglobulinemia

\section{Introduction}

Gamma heavy chain disease (gHCD) is also called Franklin's disease after the author of the first report in 1964. The disease is defined as a neoplasm of lymphocytes, plasmacytoid lymphocytes and plasma cells characterized by the production of an abnormally truncated gamma heavy-chain protein that lacks associated light chains. Since Franklin first reported the case fewer than 200 cases have been described in the literature. A wide variety of disorders have been associated with gHCD. In some cases gHCD occurs with other lymphoid neoplasms or with a history of autoimmune disorder. gHCD shows a wide clinical spectrum ranging from completely asymptomatic to progressively malignant forms.

Normal heavy chains not associated with light chains have not been detected in serum of healthy individuals. In gHCD abnormally short truncated heavy chains can be isolated from patient's serum without associated light chains. In several instances where the gene encoding the shortened heavy chain gene has been characterized the truncation originated from deletions and /or insertions within the rearranged variable region genes $(\mathrm{V})$ that in addition harbor somatic point mutations. One possibility is that several types of oncogene translocations happen as a byproduct of somatic hypermutation [1]. It has been established the gHCD immunoglobulins are the products of aberrant biosynthesis eg deletions, splice site correction and amino-terminal proteolysis [2]. Structural analysis of the defective gamma heavy chain in 23 patients showed some common features. In most cases the variable region sequence was short and interrupted by large deletions, usually including the $\mathrm{C}_{\mathrm{H}} 1$ domain. Normal sequence began at the hinge region or at the $\mathrm{C}_{\mathrm{H}} 2$ domain [3].

Genetic subtypes of multiple myeloma (MM) have been identified which have different biological features and heterogeneity in clinical outcomes [4]. New gene sequencing techniques have yielded new insights into the pathogenesis and evolution of the disease and potential individualized treatment by novel targeted approaches [5].

We report two cases of gHCD; one in a patient with lymphadenopathy and a second case in a patient with a history of

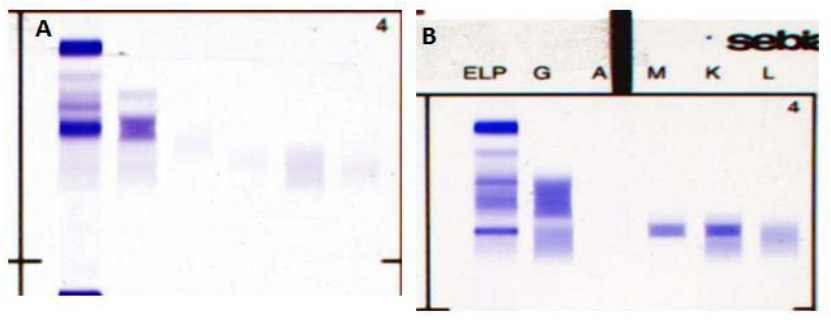

Figure 1: Serum electrophoresis and immunofixation.

(a) Case 1. Immunofixation shows staining in the gamma region, without a corresponding stain in the light chain region. Two bands are detected in the gamma region, a major and a second minor band.

(b) Case 2: Immunofixation shows a broad stain in the alpha-beta region, without a corresponding region in the light chain. Immunofixation further identifies an IgMkappa paraprotein.

ELP: Protein Electrophoresis; G: Immunofixation with anti-gamma chain identifying gamma heavy chain; A: Anti-alpha chain immunofixation; M: Anti-mu chain immunofixation; K: Anti-kappa chain immunofixation; L: Antilambda chain immunofixation

Waldenstrom's macroglobulinemia (WM). Further we review previous case series on gHCD. These two cases were diagnosed using the techniques of capillary zone electrophoresis, immunofixation and 'HevyLite' measurement. In one patient there was no evidence of previous chemotherapy. The second patient developed gHCD during treatment for WM.

\section{Case Presentation}

\section{Case 1}

A88 year old woman presented with a change in bowel habit. A colonoscopy showed some diverticular disease. A CT scan showed splenomegaly and some lymphadenopathy particularly in the region of the splenic hilum. Liver, kidney, pancreas and adrenals were normal. She had a past history of osteopenia, type II diabetes and fragility fracture. She was taking vitamin B12, vitamin D and bisphosphonates. There was no history of sweating, weight loss, bruising or recent infections. Her biochemistry and hematology at diagnosis and 3 months post-diagnosis are summarized in Table 

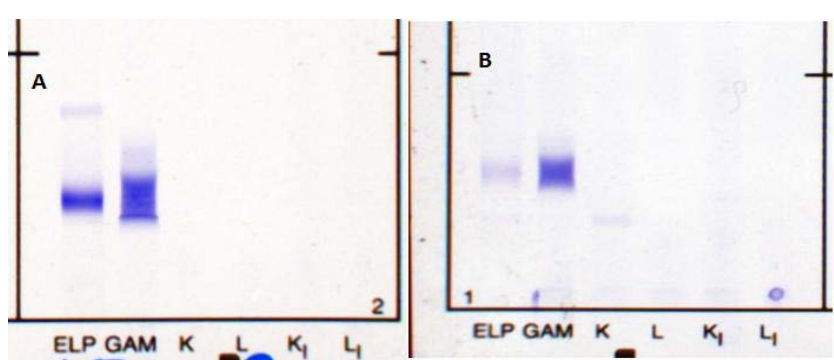

ELPAM $K$ L $K_{1} L_{1}$

Figure 2: Urine electrophoresis and immunofixation.

(a) Case 1: Urine electrophoresis revealing a broad band and a minor band in the heavy chain region, without a corresponding band in the light chain region.

(b) Case 2: Urine electrophoresis reveals a broad band in the heavy chain region without a corresponding band in the light chain region. A further kappa light chain band is observed, corresponding to the IgMkappa band identified in the serum.

ELP: Reference track showing sample proteins, GAM: Trivalent antiserum against gamma, alpha and mu heavy chains. $K$ and $L$ antisera against bound kappa and lambda light chains. $K_{1}$ and $L_{1}$ antisera against free kappa and free lambda light chains.
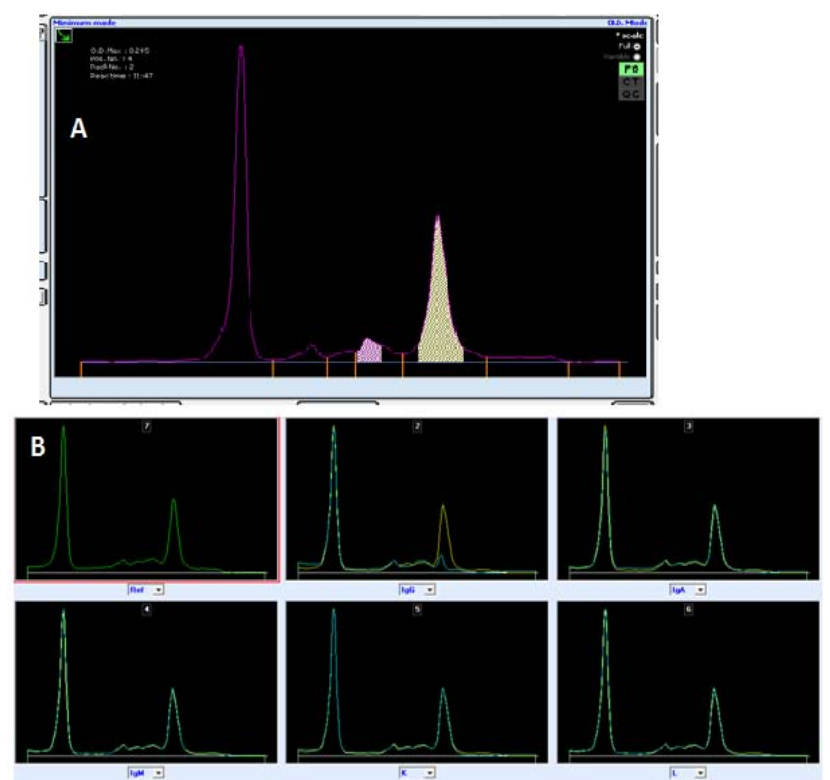

Figure 3: (a) Capillary zone electrophoresis showing a peak in the beta1 region, and a minor peak in the alpha region.

(b) Immunotyping shows a subtraction by the anti-lgG antibody, without a corresponding region in the light chain region, identifying a gamma chain peak.

Ref: Protein electrophoresis. IgG: Immunosubtraction with anti-IgG antibody; IgA: Immunosubtraction with anti-IgA antibody; IgM: Immunosubtraction with anti-IgM antibody; K: Immunosubtraction with anti-kappa antibody; L: immunosubtraction with anti-lambda antibody.

\section{$1 \mathrm{~A}, 1 \mathrm{~B})$.}

Gel protein electrophoresis and immunofixation (Figure 1A) and capillary zone electrophoresis and immunotyping (Figure 3A, 3B) (Sebia UK) identified 2 gamma heavy chains. Both methods were negative for kappa and lambda light chains. Differing sensitivities of heavy and light chain reagents can cause false negative results for light chain immunofixation and the results were confirmed by a second gel electrophoresis (Helena UK) method. Urine immunofixation

\section{Paraprotein levels during treatment}
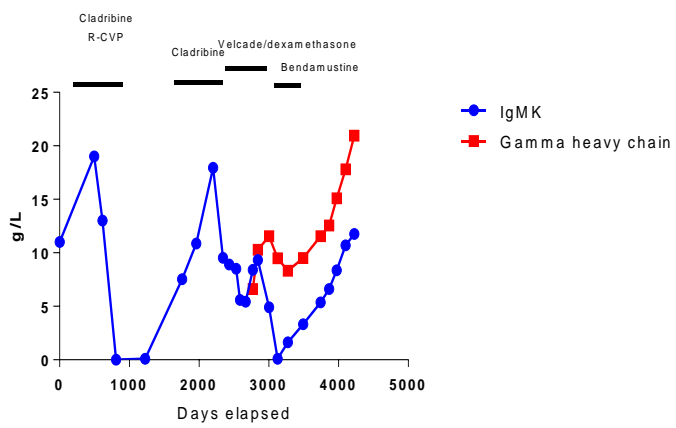

Figure 4: Case 2: Paraprotein levels during treatment.

Hospital records for the patient date back to time $=0$, post chemotherapy. $\mathrm{R}-\mathrm{CVP}=$ Rituximab with cyclophosphamide, vincristine and prednisolone.
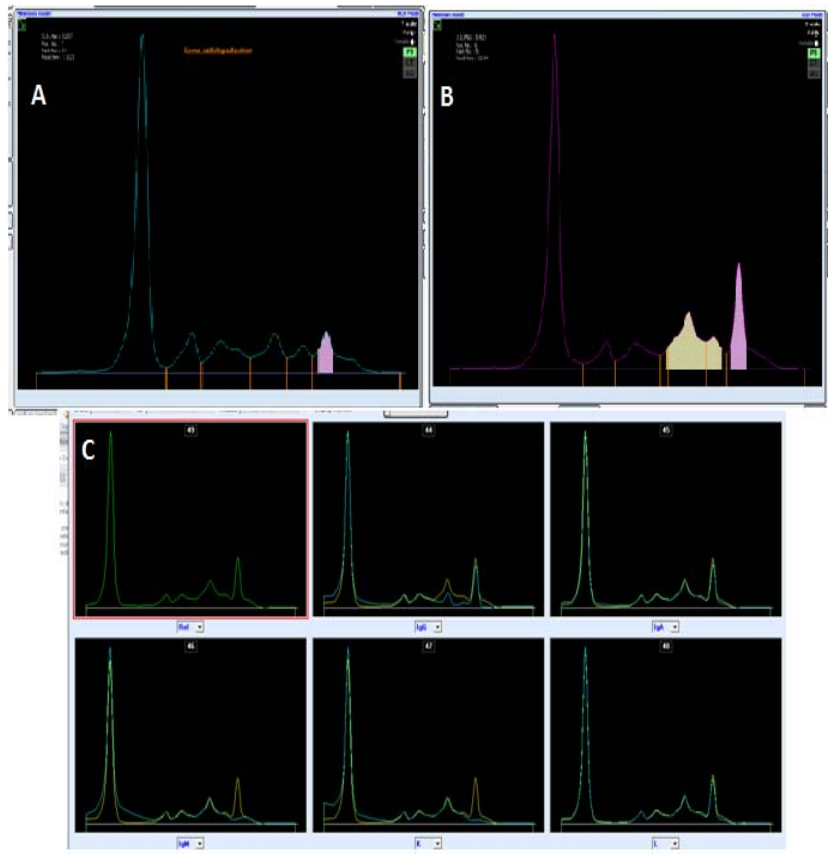

Figure 5: (a) Capillary zone electrophoresis prior to the appearance of the gamma heavy chain. A peak identified as the IgMkappa protein is detected in the gamma region

(a) Capillary zone electrophoresis identifies a broad peak in the beta region, identified as gamma heavy chain by immunotyping.

(b) Immunotyping identifies a gamma heavy chain without a corresponding light chain and an IgMkappaparaprotein.

identified a gamma heavy chain (Figure 2A).

Total IgG was elevated at $38.7 \mathrm{~g} / \mathrm{L}$ (RR 7-16), with decreased levels of IgA and IgM (Table 1A). IgG1 subclass levels were elevated, other subclass levels were lower than the reference range (Table 1E). Serum free light chain ratios were within the reference range (Table 1D). HevyLite measurements are specific for individual heavy chains with either kappa or lambda light chains and can provide more specific information than individual immunoglobulin quantitation. Observed increased heavy chain pair ratios (egIgMkappa/IgMlambda) can be indicative of clonal expansion. IgGkappa/IgGlambda ratios were within reference range confirming the absence of clonal expansion of IgGkappa/IgGlambda intact immunoglobulins (Table 1C). 


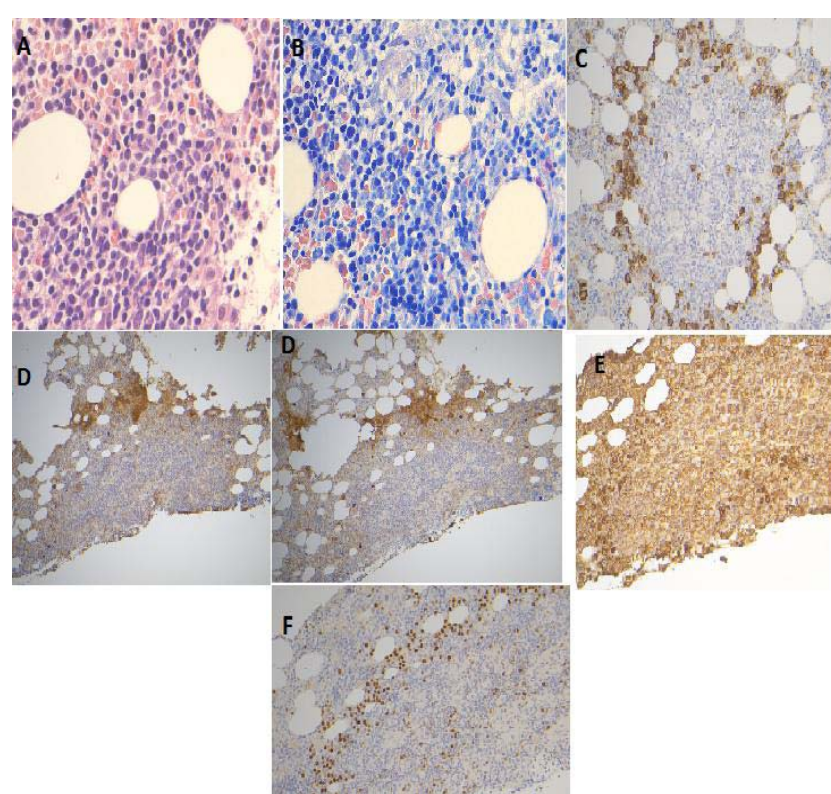

Figure 6: (a) Giemsa stain

(b) Small lymphocytes and scattered unusually small plasma cells.

(c) CD138: plasma cells forming a characteristic corona around a lymphoid nodule.

(d) (i) Lambda stain-hardly any positive cells around the lymphoid nodule.

(ii) Kappa stain-hardly any positive cells around the lymphoid nodule.

(e) IgG stain-a rim of strongly staining plasma cells.

(f) Same area stained with stained with MUM1 marker. Showing there are $\operatorname{lgG}(+)$, kappa(-), lambda(-) cells.

Bone marrow biopsy showed subtle infiltrates by low grade B-NHL, associated with groups of clonal plasma cells, which expressed neither kappa or lambda chain but stained with IgG heavy chain (Fig 6A-6F). Lymphatic infiltrate was surrounded by a rim of CD138+ plasma cells. Though there was equal expression of kappa and lambda light chains, the sum of kappa and lambda positive cells appeared to be much less than the number of CD138+ cells which indicated that most plasma cells do not express light chains.

The patient is currently monitored on a 3 monthly basis.

\section{Case 2}

Patient 2 is a 81 y old lady under treatment for WM (IgMkappaparaprotein). Details of her treatment are given in Fig 4.Capillary zone electrophoresis prior to the development of gHCD is shown in Figure 5A. Gamma heavy chain developed during treatment and an unusually diffuse gamma heavy chain band was identified by gel (Figure 1B) and capillary zone electrophoresis (Figure 5B,5C) (Sebia UK) and confirmed by a second gel electrophoresis method (Helena UK). Urine immunofixation identified gamma heavy chain and kappa light chain (Figure 2B).

Her biochemistry and hematology prior to the development of gHCD and during follow up are given in Table 1A,1B. HevyLite measurements confirmed an IgMkappa paraprotein, ratios of IgGkappa/IgGlambda were within the reference range, confirming increased IgG measurements were due IgG heavy chain (Table 1C). Serum free light chain ratio was elevated secondary to the presence of IgMkappaparaprotein (Table 1D). Her total IgG levels were

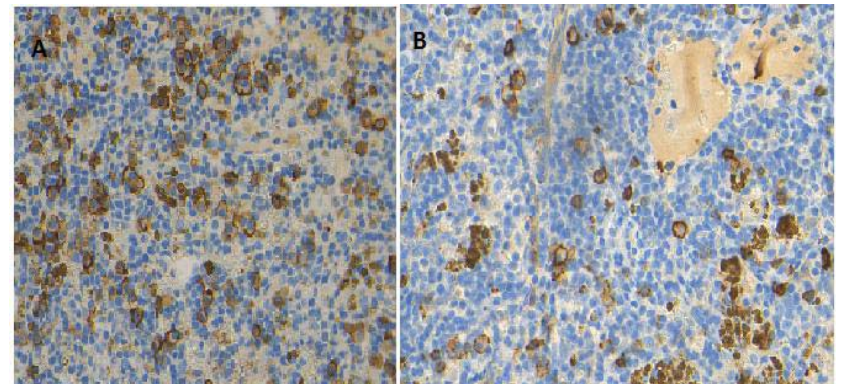

Figure 7: Bone marrow: case 2.

(a) CD138 highlight plasma cells - here admixed with lymphocytes (which are CD138 negative) Note also granular golden-brown haemosiderin deposits. (b) CD56 - some plasma cells are positive; this is an interesting finding, as plasma cells of lymphoplasmacytic lymphoma usually do not express this marker. It indirectly suggests that the 'other clone' may be more of plasma cell myeloma-like (myeloma-like MGUS). Haemosiderin which is naturally yellow/ brown is present as well.

increased at $35.1 \mathrm{~g} / \mathrm{L}$, at diagnosis of gHCD, with suppressed IgA and an IgMkappa paraprotein of $10.7 \mathrm{~g} /($ Table $1 \mathrm{~A}, 1 \mathrm{~B})$. IgG1 subtype was elevated, other $\operatorname{IgG}$ subtypes were either decreased or at the lower reference range (Table $1 \mathrm{E})$. She is currently under observation for Ibrutinib treatment,

Her bone marrow results are shown in Figure 7A,B and in the supplementary material available from the author. Lymphoma cells were CD5-, CD20+, and partially CD79a+, CD10+, CD23+, CD56+ and CD138+. A second CD56+ plasma cell clone most likely to be the gamma chain producer was identified. Bone marrow histology identified lymphoplasmacytic lymphoma/WM, which appeared IgM/kappa+ plus another population(s) of scattered plasma cells, occasionally expressing IgG and/or lambda.

\section{Discussion}

Heavy chain diseases are rare B-cell lymphoproliferative disorders that are characterized by the production of immunoglobulin heavy chains without associated light chains. gHCD has a variety of clinical presentations. The Mayo Clinic reported a series of 23 patients of whom $70 \%$ had an underlying lymphoplasma cell proliferative disorder, $13 \%$ an autoimmune disorder and $4 \%$ no underlying disease. Pathological diagnosis of underlying disease reported in the 23 patients were: lymphoplasma cell proliferative disorder, lymphoma, chronic lymphocytic leukemia, plasmacytoma, and plasma cell proliferative disorder. The disease was associated with lymphadenopathy (34\%), hepatomegaly $(4 \%)$, splenomegaly $(30 \%)$ or bone marrow involvement (30\%). The median survival of the 23 patients was $7.4 y$ (range 1 month to more than 21 years) [6]. In a further series of 13 patients, 8 patients showed a lymphoplasmacytic neoplasm that was difficult to classify. In the remaining 5 patients biopsy identified well defined neoplasms: splenic diffuse red pulp and small B-cell lymphoma, splenic marginal zone lymphoma, MALT lymphoma, lymphoplasmacytic lymphoma/WM, chronic NK-cells lymphocytosis. In the 13 cases the study reported the involvement of lymphnodes $(n=7)$, spleen $(n=2)$, bone marrow $(n=8)$ and other extranodal tissue $(n=3)$. Patients showed a female predominance (85\%) with frequent occurrence of autoimmune disease (69\%) [7]. Iijima, et al. [8] report a case gHCD and T-cell large granular 
Table 1A: Hematology and biochemistry results.

\begin{tabular}{|c|c|c|c|c|c|c|}
\hline Date & $\begin{array}{c}\mathrm{Hb} g / \mathrm{L} \\
(\mathrm{RR} 115-164)\end{array}$ & $\begin{array}{l}\text { WBC } \\
10^{9} / \mathrm{L} \\
(4-11)\end{array}$ & $\begin{array}{c}\text { Platelet } \\
10^{9} / \mathrm{L} \\
(150-400)\end{array}$ & $\begin{array}{c}\text { Neutrophils } \\
10^{9} / \mathrm{L} \\
(2-7.5)\end{array}$ & Creatinine $\mu \mathrm{mol} / \mathrm{L}$ (RR 44-80) & $\begin{array}{c}\text { Calcium } \\
\text { mmol/L } \\
\text { (RR 2.2-2.6) }\end{array}$ \\
\hline \multicolumn{7}{|c|}{ Case 1} \\
\hline At diagnosis & 115 & 2.5 & 100 & 1.6 & 46 & 2.41 \\
\hline 3 months following diagnosis & 116 & 2.8 & 101 & 2.1 & 61 & 2.33 \\
\hline \multicolumn{7}{|c|}{ Case 2} \\
\hline Post Cladribine at time $=0$ days & 120 & 5.0 & 239 & 3.1 & 71 & \\
\hline At 10 year follow up and treatment & 89 & 3.4 & 133 & 2.0 & 65 & 2.41 \\
\hline
\end{tabular}

Case 2: Records date back to time $=0$, post chemotherapy

Table 1B: Monoclonal protein studies.

\begin{tabular}{|c|c|c|c|c|c|c|c|}
\hline Date & $\begin{array}{l}\text { Total protein g/L (RR } \\
60-80)\end{array}$ & $\begin{array}{l}\text { Albumin } \\
\text { g/L (RR 35- } \\
\text { 50) }\end{array}$ & Monoclonal Band $1 \mathrm{~g} / \mathrm{L}$ & Monoclonal band $2 \mathrm{~g} / \mathrm{L}$ & $\begin{array}{l}\operatorname{Ig} G / L \\
\text { (RR } \\
7-16)\end{array}$ & $\begin{array}{l}\lg A g / L \\
\text { (RR } 0.7- \\
4.0)\end{array}$ & $\begin{array}{c}\operatorname{IgM}(\mathrm{RR} \\
0.4-2.3)\end{array}$ \\
\hline At diagnosis & 60 & 33 & $\begin{array}{c}2.8 \\
\begin{array}{c}\text { (type: gamma heavy chain in } \\
\text { the alpha region) }\end{array}\end{array}$ & $\begin{array}{c}18.5 \text { (gamma heavy chain in } \\
\text { the beta region) }\end{array}$ & 38.7 & 0.19 & 0.26 \\
\hline $\begin{array}{c}3 \text { months following } \\
\text { diagnosis }\end{array}$ & 63 & 33 & 3.21 & 21.17 & 39.7 & 0.15 & 0.24 \\
\hline \multicolumn{8}{|l|}{ Case 2} \\
\hline At time $=0$ & 78 & 39 & 11.0 (IgMK) & & 11.6 & 0.8 & \\
\hline $\begin{array}{l}\text { At } 10 \text { year follow } \\
\text { up, after several } \\
\text { treatments }\end{array}$ & 76 & 40 & $\begin{array}{l}10.7 \\
(\operatorname{lgMK})\end{array}$ & $\begin{array}{c}17.8 \\
\text { (gamma heavy chain) }\end{array}$ & 35.1 & 0.06 & \\
\hline
\end{tabular}

Records on the patient date back to time $=0$, post chemotherapy

Table 1C: HevyLite protein studies.

\begin{tabular}{|c|c|c|}
\hline Heavy chain type & Case 1 & Case 2 \\
\hline IgGKapp (3.84-12.07 g/L) & 0.99 & 1.92 \\
\hline IgGLambda (1.91-6.74 g/L) & 0.54 & 1.45 \\
\hline IgG K/L ratio (1.12-3.21) & 1.83 & 1.32 \\
\hline $\operatorname{lgA} K(0.57-2.08 \mathrm{~g} / \mathrm{L})$ & 0.23 & $<0.02$ \\
\hline $\operatorname{lgAL}(0.44-2.04 \mathrm{~g} / \mathrm{L})$ & 0.12 & 0.03 \\
\hline IgA K/L ratio $(0.78-1.94)$ & 1.92 & Not available \\
\hline $\mathrm{IgMK}=0.27 \mathrm{~g} / \mathrm{L}(0.19-1.63 \mathrm{~g} / \mathrm{L})$ & 0.27 & 11.92 \\
\hline $\operatorname{lgML}=0.04 \mathrm{~g} / \mathrm{L}(0.12-1.01 \mathrm{~g} / \mathrm{L})$ & 0.04 & 0.14 \\
\hline $\operatorname{lgM} \mathrm{K} / \mathrm{L}(1.18-2.74)$ & 6.75 & 85.1 \\
\hline
\end{tabular}

Table 1D: Serum free light chains.

\begin{tabular}{|l|c|c|}
\hline Light chain type & Case 1 & Case 2 (at time 0) \\
\hline Kappa ( $3.3-19.4 \mathrm{mg} / \mathrm{L})$ & 18.47 & 33.94 \\
\hline Lambda $16.60 \mathrm{mg} / \mathrm{L}(5.7-26.3 \mathrm{mg} / \mathrm{L})$ & 16.8 & 18.77 \\
\hline Kappa/Lambda ratio $(0.26-1.65)$ & 1.113 & 1.808 \\
\hline
\end{tabular}

lymphocytic leukemia. gHCD can exist in the absence of clinical symptoms similar to other forms of monoclonal gammopathy of undefined significance (MGUS) [9].

In our patients two methods, capillary zone electrophoresis and gel electrophoresis identified a gamma heavy chain with no corresponding light chain. HevyLite measurement confirmed the presence of gamma heavy chain in the absence of associated kappa or lambda light chains. Serum IgG subtypes showed an increase in IgG1 subclass in both patients. Gamma heavy chain was identified
Table 1E: IgG subclasses.

\begin{tabular}{|l|c|c|}
\hline & Case 1 & Case 2 \\
\hline IgG1 (RR 3.8-9.29 g/L) & 18.62 & 27.16 \\
\hline IgG2 (RR 2.42-7.0 g/L) & 0.26 & 2.92 \\
\hline IgG3 (RR 0.22-1.76 g/L) & 0.41 & 0.17 \\
\hline IgG4 (0.04-0.86 g/L) & $<0.03$ & 0.12 \\
\hline
\end{tabular}

in the urine.

Alpha, gamma and mu heavy chain diseases have been described. Alpha chain disease, the most common of heavy chain diseases, appears to be a condition affecting primarily the secretory IgA system and mainly the digestive tract. Alpha HCD may present as a continuous sequence of events from an apparently reversible hyperplastic process to an overt neoplastic proliferation [10]. $\mathrm{Mu}$ heavy chain disease is the least common of the heavy chain diseases. Patients present with lymphoplasma cell proliferative disorder, and index of suspicion is high in patients with vacuolated plasma cells in the bone marrow [6].

The mechanism for the pathogenesis of gHCD is unclear. Bone marrow histology of the first patient with isolated gHCD suggested the presence of a clonal plasma cell which selectively secreted gamma heavy chain in the absence of kappa or lambda light chain. Genomic events that underlie the progression of MGUS to MM have been difficult to dissect. Both bone marrow microenvironment and genomic instability play a role in MM pathogenesis. Bone marrow biopsy in the second patient, with WM and gHCD identified two possible malignant clones. It is worth noting that this patient's treatment included R-CVP, cladribine and velcade. It is plausible that 
the treatment might have increased the transformation of the gHCD clone by increasing the instability of the MM cells. Clonal evolution in MM before and after therapy can follow several patterns. Branching clonal evolution involves one or more subclones that emerge at a later time point. Current therapeutic approaches have shortcomings, and understanding of MM tumorogencsis and progression may have implications for genomically guided precision treatment [11].

\section{Conclusion}

In summary we report two cases of gHCD. In the one case gHCD developed as the presenting lymphoid malignancy. Bone marrow biopsy revealed an IgG heavy chain secreting clone. The second case was a composite lymphoid neoplasm, of WM and gHCD. gHCD developed during treatment for WM. Bone marrow biopsy revealed the presence of two neoplastic lymphoid cell lines. Review of the literature suggests further studies are indicated to develop an understanding of the clonal evolution of gHCD.

\section{References}

1. Goossens T, Klein U, Küppers R. Frequent occurrence of deletions and duplications during somatic hypermutation: implications for oncogene translocations and heavy chain disease. Proc Natl AcadSci 1998; 95: 2463 2468.

2. Alexander A, Anicito I, Buxbaum J. Gamma heavy chain disease in man Genomic sequence reveals two noncontiguous deletions in a single gene. J Clin Invest. 1988; 82: 1244-1252.
3. Wahner-Roedler DL, Kyle RA. Heavy chain diseases. Best Pract Res Clin Haematol. 2005; 18: 729-746.

4. Sawyer JR. The prognostic significance of cytogenetics and molecular profiling in multiple myeloma. Cancer Genet. 2011; 204: 3-12.

5. Jiménez C, Jara-Acevedo $M$, Corchete LA, Castillo D, Ordóñez GR, Sarasquete ME, et al. A Next-Generation Sequencing Strategy for Evaluating the Most Common Genetic Abnormalities in Multiple Myeloma. J Mol Diagn. 2017; 19: 99-106.

6. Wahner-Roedler DL, Witzig TE, Loehrer LL, Kyle RA. Gamma-heavy chain disease: review of 23 cases. Medicine (Baltimore). 2003; 82: 236-250.

7. Bieliauskas S, Tubbs RR, Bacon CM, Eshoa C, Foucar K, Gibson SE, et al. Gamma heavy-chain disease: defining the spectrum of associated lymphoproliferative disorders through analysis of 13 cases. Am J Surg Pathol. 2012; 36: 534-543.

8. lijima M, Sekiguchi N, Nagat A, Wagatsuma M, Midorikawa K, Kurimoto M, et al. Gamma heavy chain disease with T-cell large granular lymphocytic leukemia: a case report and review of the literature. Intern Med. 2016: 55: 399-403.

9. Van Keer J, Meijers B, Delforge M, Verhoef G, Poesen K. Two cases of heavy chain MGUS. Case reports in Oncological Medicine. 2016.

10. Seligmann M. Alpha-chain disease. J Clin Pathol Suppl (AssocClinPathol). 1975; 6: 72-76.

11. Bahlis NJ. Darwinian evolution and tiding clones in multiple myeloma. Blood. 2012; 120: 927-928.
Ann Hematol Oncol - Volume 5 Issue 3 - 2018

ISSN : 2375-7965 | www.austinpublishinggroup.com

Ramasamy et al. (C) All rights are reserved
Citation: Ramasamy I and Rudzki Z. Gamma Heavy Chain Disease: 2 Cases. Ann Hematol Oncol. 2018; 5(3): 1198. 